\title{
Paysage cadastral et paysage vécu: réflexions sur les espaces construits
}

Une idée volontiers reçue dans les milieux d'aménagistes est que l'espace bâti - et donc, pour une part de plus en plus large, l'espace urbain - n'occupe encore, en Suisse, que peu de terrain. Les mesures faites au sol justifient, incontestablement, cette façon de voir.

Un premier relevé, qui remonte à la publication par le Bureau fédéral de statistique, en 1952, d'une "statistique de la superficie de la Suisse» n'accorde que $2 \%$ du pays aux «fondements des constructions, cours des maisons et parcs». Il ne s'agit pas d'un inventaire exhaustif: seules les communes entièrement cadastrées - elles couvrent alors, en étendue, un peu plus du quart de la Confédération - ont été prises en comptel $)$. Quoique partielle, cette mesure livre pourtant déjà de précieuses informations. Elle indique une limite supérieure de la surface bâtie, car parmi les 1272 communes en question, figurent pratiquement toutes les villes de plus de 10.000 habitants et la majorité de celles de plus de 5.000 .

Une seconde recension, plus complète mais moins précise, nous vient des "Vade-mecum» annuels publiés par l'Institut ORL. L'édition de 1973/74 estime la «surface nette d'habitation» à $2,5 \%$ pour l'ensemble de la Suisse ${ }^{2}$ ). L'écart entre les deux valeurs extrêmes - $0,5 \%$ dans les Grisons, $62 \%$ à Bâle-ville - se comprend aisément. Au total, cependant, les variations autour de la moyenne restent modérées. Elles se tiennent entre 1 et $2 \%$ pour les cantons de montagne; elles montent à $4 \%$ dans les cantons de la «Bandstadt» (SG, TG, AG, SO, NE); elles atteignent $8 \%$ dans le canton de Zurich et culminent à $13 \%$ dans celui de Genève (BS, déjà nommé, étant excepté). Les terrains réservés aux immeubles ont donc gagné en étendue, entre 1950 et 1970, d'environ $25 \%$. Ce qui correspond à l'accroissement démographique de cette période. On s'étonne que la progression n'ait pas été sensiblement plus marquée du côté du domaine bâti. Quoiqu'il en soit, et quand bien même de nouvelles évaluations conduiraient à les doubler, ces pourcentages sont modestes. Dans les parties les plus densément peuplées du Plateau la marée des maisons conserve donc un caractère graduel ${ }^{3}$ ). L'objectivité froide des chiffres ne cadre pas avec l'inquiétude montante de bien des milieux. Face à la poussée urbaine, on en vient à poser le problème en termes de choix cruciaux à l'égard de la croissance. Où donc est la vérité?
Nous aimerions montrer, dans les lignes qui suivent, qu'une comptabilité cadastrale du sol bâti, aussi exacte soit-elle, ne restitue, de toute manière, qu'un aspect de la réalité. Le paysage construit est, dans l'image effective qu'en reçoivent les habitants et dans l'interprétation qu'ils en donnent, plus étendu que ne l'est l'emprise au sol des immeubles; et, qualitativement, différent de ce que suggère la distribution, vue en plan, des maisons. Or, la prise en considération du comportement humain s'impose absolument à qui veut saisir la réalité dans la pluralité de ses dimensions: ce qui est, par vocation, le cas des représentants des sciences humaines aussi bien que des responsables de l'aménagement du territoire.

\section{A. Logique du paysage vécu}

L'environnement bâti, tel qu'il est vécu par les hommes, réunit, nous semble-t-il, quatre propriétés fondamentales.

I. La première est sa très large extension. Le paysage urbain tend à gagner tous les espaces construits, y compris ceux qui ne répondent pas à la définition de «ville», au sens strict. Dans nombre de localités rurales les signes architecturaux de citadinisation se multiplient. Le visage traditionnel du village subit un remodelage sensible partout, notamment là où une fraction de la population résidante prend part aux migrations alternantes de travail. En 1970, 0,3\% des communes suisses seulement échappaient encore aux mouvements pendulaires. La statistique des constructions recoupe l'impression d'urbanisation des campagnes qu'impose l'observation directe. On peut estimer, sur la base des renseignements partiels qu'elle fournit, que s'érigent, en moyenne chaque année, 5.000 à 10.000 immeubles nouveaux pour l'ensemble des localités de moins de 2.000 âmes (entre 1950 et 1970). Il ne fait pas de doute que ces constructions revêtent des aspects extérieurs qui les apparentent étroitement à l'habitat, aux usines et aux immeubles de service des centres urbains. Elles répondent, fondamentalement,

Prof. Dr. J.-L. Piveteau, Institut de géographie, Université de Fribourg, 1700 Fribourg 
aux besoins d'ouvriers ou d'employés. Aussi, lorsqu'on se déplace, quotidiennement pour des raisons professionnelles, hebdomadairement pour des motifs de détente, à travers la campagne helvétique, on fait l'expérience d'un environnement construit qui, aux dimensions des groupements près, revêt une livrée de moins en moins spécifiée, alignée qu'elle est sur le style urbain. La phase de développement des villages dans le sens non-agricole qui vient d'être indiqué, correspondant, pour l'essentiel, au dernier quart de siècle, ces multiples fronts ruraux de croissance immobilière différent moins, physionomiquement, des couronnes urbaines, leurs contemporaines, qu'ils ne se distinguent, comme ces dernières $d u$ reste, de leurs centres respectifs.

II. La perception de l'environnement bâti excède largement la stricte emprise au sol des constructions. Et cela pour deux raisons principalement.

D'abord les hommes vivent à ras du sol. Les utilisateurs de cartes que sont les géographes et les aménagistes risquent parfois d'oublier cette vérité première. Les paysages se présentent à l'habitant par la tranche, rarement selon une perspective cavalière, jamais à la verticale. Autre évidence, nécessaire à rappeler: un immeuble, même modeste, se voit de plusieurs centaines de mètres. Ce qu'on pourrait appeler, par analogie avec le phénomène climatique connu, "l'ombre portée bâtie», est l'impression produite, à distance, par des constructions sur un observateur quelconque, sur l'homme de la rue. Cette ombre portée se révèle très supérieure à la surface effectivement occupée par les immeubles (cf. fig. 1 à 4). Elle s'accroît avec la taille de ceux-ci. Les rémissions que constituent les périmètres non occupés par des maisons à l'intérieur d'une zone construite (jardins, places, terrains vagues, voire prés ou champs) sont appréciées comme une caractéristique d'aération du tissu villageois ou citadin. Elles n'effacent pas l'attribut «bâti» de l'environnement en cause.

L'insertion d'une localité dans un contexte accidenté, une vallée par exemple, peut avoir un effet «ruralisant», dans la mesure où la personne qui se déplace aperçoit en arrière-plan, même si cela se fait par bribes, des étendues encore couvertes de forêts ou de cultures. Une topographie de plaine n'offre pas d'avantages semblables. Mais il arrive aussi qu'à cause du relief précisement, on découvre, à chaque brèche ouverte par une rue transversale, des quartiers d'habitation accrochés aux versants encadrants. Les sites plats prennent ici leur revanche, puisqu'ils dissimulent de telles extensions.

Le rôle du mécanisme de l'ombre portée nous paraît particulièrement important dans le cas des centres ruraux. Car pour peu, comme nous le notions précédemment, que ceux-ci comprennent plusieurs maisons à caractère urbain, l'impression produite sur l'habitant (ou le transitant) ne correspondra pas, mais par excès, à ce qui est effectivement construit: ce sera un sentiment probable d'urbanisation, que ne percevra pas, quant à lui, le simple lecteur, sur plans, de la réalité.

La seconde raison est que citadins ou villageois empruntent, dans leurs déplacements professionnels ou privés, les axes ferroviaires ou routiers les plus directs ou les mieux desservis. Ceux-ci se trouvent avoir fixé, pour cette raison même, résidences, entreprises et services. Il ne s'agit, certes, assez souvent, que d'un rideau de constructions, sans véritable profondeur de part et d'autre de l'itinéraire suivi. Le cas se rencontre dans les campagnes à villagesrue ou à villages de colonisation forestière; mais également dans la périphérie de bien des villes, aux axes de développement, encore aujourd'hui, fréquemment digités. Il n'empêche que, pour une aire bâtie donnée, les cheminements effectués par les hommes démultiplient leur univers de logements, de magasins et d'usines.

III. L'importance de l'environnement bâti dépend de la fréquence des déplacements humains. Un objet se remarque d'autant mieux s'il se déplace. De même, perçoit-on davantage le cadre immobilier, immuable par essence, à la faveur de sa propre mobilité.

Le rythme et l'envergure des circuits journaliers vont grandissant. Les migrations alternantes liées au travail et aux loisirs, la motorisation accélérée, provoquent ou facilitent, allongent en tout cas les trajets. Les hommes traversent aujourd'hui, quotidiennement, notablement plus d'espaces construits que naguère. L'image urbaine occupe une place accrue dans la mémoire visuelle de chaque habitant. Plus les hommes parcourent un territoire, plus les aspects de celui-ci - c'est-à-dire son paysage, quel 
qu'il soit, mais, en l'occurrence et surtout, le paysage construit -, sont «utilisés», vécus, même si le type d'appréhension ne correspond vraisemblablement jamais à une observation dans le sens où l'entendent soit les artistes, soit les scientifiques.

Par ailleurs, plus il y a d'hommes à subir - ou à goûter - l'environnement bâti, plus l'urbanisation doit être tenue pour importante. Il nous paraît en effet juste de complèter la notion aréolaire d'urbanisation, définie à l'aide de différents paramètres mais sur la base des seules étendues construites, par l'idée que l'accroissement des densités humaines comme celui des «vibrations» pendulaires, en multipliant les contacts entre l'homme et son cadre, confèrent du même coup une autre dimension du phénomène. L'urbanisation devient volume. On s'est soucié exclusivement de la première perspective jusqu'à présent. Une vision globale de la réalité devrait y inclure la seconde. L'analogie entre le concept élargi d'urbanisation, tel que nous le proposons, et le concept de puissance économique régionale, vient spontanément à l'esprit. On définit en effet couramment cette dernière comme étant le produit du revenu national par tête (propre à la région analysée) par le nombre d'habitants de la région en question. Sans doute la perception de l'environnement ressortit-elle à la subjectivité. Mais la prise en compte de cette subjectivité relève, quant à elle, de l'objectivité.

Il y aurait, ainsi, trois niveaux d'appréciation de l'environnement bâti - urbain ou en passe d'urbanisation.

Le premier, que nous qualifierons de "cadastral», s'en tient à une comptabilisation stricte de l'emprise au sol, des différentes constructions qui s'y trouvent fixées.

Le second, que nous appellerons «virtuel», s'établit sur la base d'un nombre conventionnel d'itinéraires - reliant, par exemple, dans un espace régional défini, tous les centres entre eux. Une certaine pondération peut ici intervenir, qui tient compte du grain du tissu bâti, de la hauteur moyenne des immeubles, et de la proportion de personnes appartenant à chacun des trois secteurs socio-économiques - ce qui, dans le cas des localités rurales en tout cas, doit s'exprimer au travers de la physionomie des constructions.

Le troisième constitue l'environnement "effectif». Il introduit dans le calcul précédent deux données supplémentaires: les rythmes de déplacement et le nombre d'habitants concernés par le périmètre étudié.

IV. Vu sous un angle dynamique, l'environnement bâti ne peut que gagner en importance et, conjointement, sinon même plus rapidement encore, le caractère urbain de cet environnement en fait autant. La démonstration est superflue. Ce qui mérite de retenir l'attention, en revanche, ce sont les éléments modérateurs du processus. On risque de les négliger. Nous en citerons trois qui nous frappent.

Les nouvelles constructions, pour une part d'entre elles, s'insèrent dans un tissu bâti déjà existant. La perception des habitants se nuance en conséquence - une densification du paysage ne peut laisser insensible -, mais elle ne se modifie pas substantiellement, car, dans le cas des villes à tout le moins, l'empreinte citadine précédait l'accroissement.

Ensuite, le rayon du drainage de main-d'œuvre pendant la semaine, celui des pulsions de loisirs le dimanche, s'allongent. Ils intègrent nécessairement davantage de paysages proprement ruraux dans le bilan des impressions reçues.

Les autoroutes, enfin, en Suisse comme dans les autres pays, proposent, en raison de leur tracé, une vision agro-sylvo-pastorale des contrées qu'elles traversent. Elles libèrent des réserves dormantes d'horizons largement affranchis de tout signe urbain.

\section{B. Quelques mesures de contrôle sur la carte}

Nous avons voulu vérifier sur quelques cas concrets deux au moins des quatre propositions qui viennent d'être avancées.

Le niveau physionomique d'urbanisation transparaît au travers de deux dénombrements: celui des types d'activités des résidents de chaque commune et celui du nombre de constructions récentes. On peut admettre que les traits «urbains» croissent en liaison étroite avec le pourcentage de gens travaillant dans les secteurs secondaire et tertiaire d'une part, comme d'autre part, avec l'érection, d'un recensement à l'autre, de nouveaux immeubles. Mais il y a là matière à longs dépouillements débouchant sur des résultats sans totale rigueur. Et si l'observation di- 
recte constitue une autre approche, techniquement plus simple et statistiquement plus précise, elle exige, en revanche, un travail de relevé sur le terrain infiniment contraignant. Nous y avons donc renoncé. Au stade exploratoire qui est le nôtre dans cette recherche, un tel investissement ne se justifie pas encore.

Quant à la mesure des trajets réels, des rythmes de fréquentation de chaque itinéraire, du nombre de personnes en mouvements quotidiens de toutes sortes, il nous a paru hors de question de l'établir, dans l'état actuel de la documentation chiffrée. Seules des enquêtes sur place, longues et minutieuses, dépassant de loin ce que nous sommes matériellement en mesure d'entreprendre, auraient permis de rassembler les informations nécessaires.

Notre propos s'est donc limité à une confrontation très approximative de la perception de l'environnement bâti et de l'emprise au sol des constructions, ainsi qu'à une esquisse d'évolution comparée de la première et de la seconde. Soit les points II et IV du chapitre qui précède.

I. Notre démarche fait usage d'un modèle, dans la mesure où nous décidons d'apprécier l'environnement bâti en additionnant les parties construites le long de tous les itinéraires qui relient le centre d'une localité repère au centre de chacune des autres localités situées à l'intérieur d'un périmètre défini.

La part de convention saute aux yeux. Les déplacements des habitants dépassent en complexité ces itinéraires symboliques. L'attractivité des différents noyaux varie dans des proportions considérables en gros, comme toujours, en fonction de la taille -. Le nombre d'habitants concernés par les mouvements échappe enfin à un tel calcul.

Il nous paraît toutefois qu'une telle méthode donne le volume minimal du paysage susceptible d'être perçu comme urbain ou en voie d'urbanisation. Sans doute les agriculteurs circulent-ils, pour une bonne part de leur temps, de leur ferme à leurs champs. Leur horizon agreste tend donc à diminuer l'impression d'un contexte bâti. Mais ils ne représentent qu'une proportion réduite de la population en général, et même au sein des villages encore "ruraux»" ne constituent-ils souvent qu'une fraction minoritaire. En considérant autour d'une localité «l» toutes les autres localités $x, y, z$, etc.... situées dans un rayon rapproché, on dispose d'une partie des lieux vers lesquels la population de «l» se déplace. Mais il ne fait pas de doute que la majorité des circuits s'effectuent, soit à l'intérieur de la localité elle-même, soit entre la localité considérée et les localités - voisines ou éloignées - les plus importantes de la région, c'est à dire celles dont, précisément, le paysage est à la fois le plus urbanisé et le plus étendu. La mesure égalitaire de centre à centre que nous avons pratiquée a, de la sorte, la valeur d'un seuil inférieur: elle se situe, selon toute vraisemblance, en-dessous de l'importance réelle de la perception de l'environnement bâti.

Nous avons opéré sur plusieurs périmètres-tests, carrés, de $5 \mathrm{~km}$. de côté. Le choix de leur emplacement relève de l'empirisme. Il repose sur le souci d'observer des cas différents quant à la taille et quant à l'implantation de la localité prise comme exemple. L'échantillonnage ne prétend à aucune rigueur statistique. Il a le caractère simplement indicatif des approches monographiques (fig. 5).

L'identification des centres relevait de l'examen clinique cartographique. En règle générale, nous avons retenu le chef-lieu de chaque commune.

Les "iitinéraires», ont été tracés à vol d'oiseau. Il s'agissait là d'un accommodement d'ordre évidemment pratique, qui ne pouvait, une fois encore, que sous-évaluer l'impression vécue par les habitants.

Tous les espaces construits - à l'exception des maisons réellement isolées - ont été enveloppés d'une ligne tracée à environ 100 mètres (ou moins) des immeubles concernés. Un groupe de maisons ou d'usines, séparé de la localité la plus proche par une distance supérieure à 100 mètres, faisait par conséquent l'objet d'une délimitation propre. Il entrait dans les mesures d'environnement bâti, non pas en tant que "centre», puisqu'il n'en était pas, mais comme zone construite, chaque fois qu'un des itinéraires de centre à centre le traversait (fig. 6).

La confrontation entre l'emprise au sol et l'environnement bâti virtuel, menée dans le cadre de nos 18 échantillons, nous conduit aux remarques suivantes.

1. L'écart est sensible entre les deux phénomènes. Du côté de l'environnement bâti, les pourcentages sont, en moyenne, dix fois supérieurs (d'après les comptages opérés sur des éditions de la carte natio- 
nale au $1: 50.000$ remontant également à la décennie des années cinquante). Si l'on admet les conventions posées et explicitées au paragraphe précédent, la différence enregistrée confirmerait donc amplement ce que la réflexion théorique suggérait tout à l'heure: l'urbanisation perceptible - ou, à strictement parler, les constructions perceptibles - dépassent de beaucoup l'espace réellement occupé par des immeubles.

2. Un autre contraste oppose «l'environnement» à «l'emprise». Pour celle-ci, l'amplitude entre les valeurs extrêmes va de 1 à 10 ; pour celui-là, la fourchette se réduit à une ouverture de 1 à 4 . Ainsi les inégalités d'encombrement immobilier, d'une région ou d'un lieu à l'autre, devraient être moins brutalement perçues par le touriste, le pendulaire ou le résident que par l'arpenteur. L'hypothèse demande à être appuyée par d'autres mesures. Elle n'en demeure pas moins très plausible, car l'habitant connaît les paysages de manière linéaire, les géomètres, les aménagistes ou les géographes, de façon essentiellement aréolaire.

3. Les évaluations pratiquées à l'intérieur de nos périmètres montrent que pour un même indice d'environnement bâti, on obtient des longueurs absolues de trajets allant du simple au double. Le nombre, variable, des centres de peuplement, explique ces différences. Cette observation conduit à proposer le principe suivant. A densité démographique régionale égale, et pour un rapport entre «itinéraires en zone construite» et «total des itinéraires disponibles» qui resterait constant, la distribution des hommes entre un plus grand nombre de localités, accroît, simultanément, l'impression d'urbanisation et celle de ruralisation. Pour l'habitant, en effet, se multiplient alors, dans les limites d'un espace donné, les virtualités de déplacements le long de rues comme à travers champs. Sous l'angle de l'appréhension vécue - possible ou réelle ${ }^{4}$ - qui est celui du présent article, l'urbanisation croissante ne s'oppose donc pas nécessairement à une "ruralisation" également croissante, aussi paradoxale qu'en paraisse, au premier abord, l'idée.

II. Pour connaître l'évolution de l'environnement bâti, nous disposions d'un document de choix: les cartes de la Suisse au $1: 25.000$, publiées régulièrement depuis une centaine d'années (celles de «l'Atlas topographique» et celles du «Service topographique fédéral»). Nous avons effectué, sur quelques-uns de nos échantillons, une confrontation diachronique, échelonnée de la manière suivante: 1898/1902, 1950/ $1955,1968 / 1970$. Les dates d'édition ou de révision, variables d'une carte à l'autre, ne permettent pas de coupe unifiée quant à l'année.

Afin d'apprécier la croissance du paysage construit là où il a le plus de chance d'atteindre des valeurs élevées, nous avons commencé par quelques secteurs de la «Bandstadt» helvétique (fig. 7).

Sans doute la convergence des résultats obtenus restera-t-elle précaire, tant qu'un plus grand nombre de mesures ne viendra pas l'étayer. Elle mérite cependant une analyse, et sans risque, puisque nous ne la tenons que pour une hypothèse de travail.

"L'environnement bâti virtuel» s'accroît selon un rythme, semble-t-il, légèrement décélérant - dans les zones déjà passablement urbanisées en tout $c a s^{5}$ ). Et cela, dans le même temps où l'emprise au sol augmente de façon exponentielle. (L'hésitation sur ce dernier point n'est pas de mise, encore qu'une évaluation précise s'avère difficile. Nous nous sommes fondé, pour l'établir, sur le nombre de «maisons habitées» aux trois recensements de 1900, 1950 et 1970.)

La lecture comparée des cartes montre que le tassement constaté dans la pousée récente de «l'environnement bâti», peut être imputé à la densification des îlots d'habitat, au comblement de certaines zones situées entre les digitations originelles d'extension. Mais il peut s'expliquer aussi par l'expansion grossièrement circulaire des centres urbains: le rayon d'un cercle ne croît en effet que comme la racine carrée de la superficie de ce cercle; en d'autres termes, la distance nécessaire à parcourir pour gagner le centre d'une ville depuis sa périphérie, augmente sensiblement moins vite que ne le fait la surface progressivement occupée par cette localité. Et, l'assertion s'applique encore mieux aux grandes agglomérations qu'aux petites. La courbe de «l'environnement bâti virtuel» a le type «logistique» si, du moins, on admet qu'une inflexion à concavité tournée vers le haut marque ses débuts. La supposition nous paraît fondée. 
Cette dernière remarque, ajoutée à celles qui la précédent, nous conduit, sous bénéfice d'inventaire, aux trois suggestions suivantes.

- Les zones construites perceptibles - virtuellement perceptibles, évidemment - ont des chances, à court et à moyen terme, de ne pas croître aussi vite que l'aire effectivement bâtie. A condition d'être replacé dans une telle perspective dynamique, donc relativiste, l'optimisme affiché par certains aménagistes trouverait là une justification.

- Le rythme de progression du contexte bâti (virtuellement perceptible) a dû, au cours du XIXème et peut-être encore au début du XXème siècle, être supérieur au rythme de progression des emplacements effectivement construits. Nous avons pu constater, en tout cas, que l'avance de «l'environnement» sur «l'emprise» demeure, présentement considérable.

- Enfin, à ce décalage actuel entre la surface occupée au sol par le complexe immobilier et la projection mentale que sont susceptibles d'en recevoir les hommes, s'ajoute un autre déphasage de perception, plus subjectif: entre les modifications effectives du paysage et la conscience qu'en prend l'habitant. Les deux distorsions demandent à être clairement distinguées. Il resterait à se demander si ce second mécanisme psychologique - qui se manifeste aujourd'hui par un certain alarmisme, peut-être abusif ne correspond pas, pour une part du moins, au franchissement d'un seuil critique de perception. Au delà de cette limite, tout accroissement de l'urbanisation, fut-il fortement décélérant, devient agression chronique.

\section{Zusammenfassung}

Die Zunahme der Urbanisierung im allgemeinen, die unmerkliche Ausdehnung der Urbanisierung in den ländlichen Gebieten im besonderen ist allen bekannt. Das Ausmaß dieses Prozesses ist jedoch schwierig einzuschätzen. Die konkreten Veränderungen der Landschaft scheinen auf den ersten Blick sichere Beweise für die Urbanisierung zu sein; aber zwischen der Abschätzung dieser Veränderungen und dem Eindruck, den die Veränderungen auf die Einwohner machen, kann eine bedeutende Diskrepanz bestehen.
Dieser Artikel vertritt die Idee, daß die Wahrnehmung der bebauten Umwelt offensichtlich rein subjektiv sei; wenn man aber die Organisation des Raumes in seiner Gesamtheit kennen lernen will, muß man diese Subjektivität in Betracht ziehen.

\section{Résumé}

La poussée de l'urbanisation en général, l'infiltration plus subtile de l'urbanisation à travers les campagnes, sont reconnues de tous. Cependant, la mesure de ces processus est délicate. Les transformations concrètes du paysage apparaissent, à première vue, comme autant de témoignages sûrs; mais entre l'évaluation qu'on peut faire de ces changements et l'impression que ceux-ci produisent sur les habitants, l'écart risque d'être important.

Cet article défend l'idée que la perception de l'environnement bâti ressortit, de toute évidence, à la subjectivité, mais que si l'on veut connaître l'organisation de l'espace dans sa totalité on doit tenir compte de cette subjectivité.

1) Les $2 \%$ ne s'appliquent, évidemment, qu'aux communes cadastrées.

2) Dans un autre document («Conceptions directrices d'aménagement du territoire national»), l'Institut ORL évalue à $2,3 \%$ la "surface nette d'agglomération». (Août 1973.)

3) On évalue la surface accaparée chaque année par les nouvelles constructions à 700 hectares, soit $0,017 \%$ du territoire national.

4) Mais il n'est question, rappelons-le, dans cette seconde partie de notre recherche, que de l'appréhension vécue possible. Une mesure «réelle» de l'urbanisation ressentie devrait prendre en compte le nombre de personnes circulant et le rythme des déplacements. (cf. parag. A. III.)

${ }^{5}$ ) Le cas de Courtepin (cf. fig. 7) suggère que les zones rurales pourraient connaître un trend sensiblement différent. 
Fig. 1-4: Schémas théoriques relatifs à la perception de l'environnement bâti.

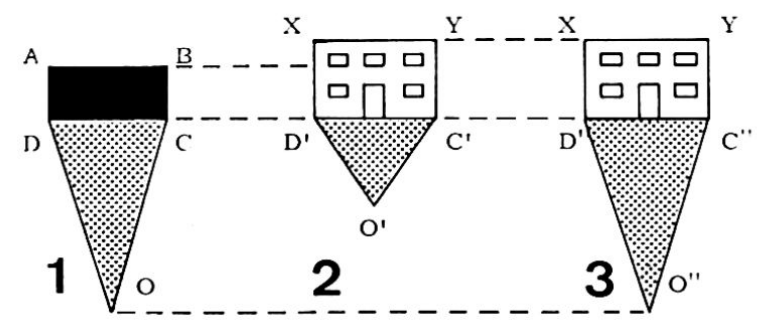

Perburbations et rectifications introduites par le réalisme visuel et le réalisme intellectuel

La figure 1 est la projection au sol de la maison, 'et de l'angle sous' lequel l'observateur la voit. L'emprise au sol de l'immeuble ABCD est inférieure a. "l'ombre portée batie" OCD que crée la présence de cet immeuble pour l' $^{\prime}$ observateur $O$.

La figure 2 représente ce que, à strictement parler, 1'observateur appréhende: d'une part la façade (le rectangle $C^{\prime} D^{\prime} X Y$ ), et, d'autre part, atténué par les lois de la perspective, l'espace qui le sépare de cette façade. Pour l'observateur (O), le triangle OCD apparaft donc, pendant un laps de temps tres bref, plus petit qu'il n'est en réalité. $D^{\prime}$ ou, sur notre schéma, $O^{\prime} C^{\prime} D^{\prime}<O C D$.
Mais, en fait, le réalisme intellectuel corrige partiellement le réalisme visuel. Il ne modifie pas l'importance accordée à la façade, car elle est vue de champ, de plein fouet, et elle dépasse généralement en dimensions l'emprise au sol de la maison elle-merme. En revanche, il tend à redonner au triangle $O^{\prime} C^{\prime} D^{\prime}$ de "l'ombreportée batie" und dimension supérieure à celle que lui assignerait une stricte impression sensorielle. L'action conjuguée d'une perception non retouchée (en ce qui concerne le plan vertical de la façade) et d'une perception, elle, au contraire rectifiée (en ce qui concerne l'espace séparant l'observateur du batiment qu'il regarde), conduit à un total non négligeable. La figure 3 résume cette situation terminale: $C^{\prime \prime} D$ " $X Y$, égal à $C D X Y$, est supérieur à $A B C D$; et $O{ }^{\prime \prime} C$ " $D$ " retrouve pres que l'étendue de $O C D$. (Les triangles $O^{\prime} C^{\prime} D^{\prime}$ et $O^{\prime \prime} C^{\prime \prime} D$ ", des figures 2 et 3 , sont évidemment situés dans un plan perpendiculaire au plan auquel appartiennent les rectangles $C^{\prime} D^{\prime} X Y$ et $C^{\prime \prime} D^{\prime \prime} X Y$; à la différence du triangle $O C D$ et du rectangle $A B C D$, de la figure 1 , qui appartiennent, eux, à un meme plan).

- Introduction de l' élément dynamique : le déplacement de l'observateur

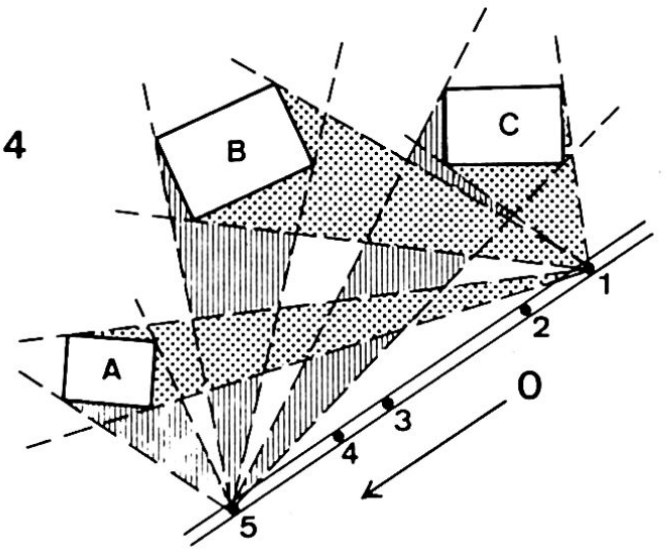

$L^{\prime}$ angle sous lequel $\mathrm{l}^{\prime}$ observateur $\mathrm{O}$ voit les immeubles $\mathrm{A}, \mathrm{B}$ et $\mathrm{C}$, change à mesure qu' il se déplace de 1 en 5 . Sans doute l'observateur ne regarde-t-il pas constamment ce qui l'entoure. Mais le total des "instantanés" qu'il a pris successivement $(1,2,3,4,5)$ équivaut à lui avoir fait balayer du regard tout l'espace compris à l'intérieur du polygone $\mathrm{A}, \mathrm{B}, \mathrm{C}, \mathrm{O}_{1}, \mathrm{O}_{5}$.

Son réalisme visuel lui fait donc tenir ce polygone entier pour "l'ombre portée bâtie", alors mé me que son réalisme intellectuel l'amène à considérer les trois bâtiments $\mathrm{A}, \mathrm{B}$, et $\mathrm{C}$ comme une constante.

Ainsi, la mobilité de l'observateur non seulement le rend attentif à la présence des éléments immobiles - le contexte est mieux vécu - mais elle accron sensiblement l'étendue relative de l'"ombre portée bâtie". 
Fig. 5: Emplacement des 18 périmètres-tests.

Fig. 6: Mode de mesure de l'environnement bâti virtuel.

Fig. 7: Evolution comparée de l'emprise au sol des immeubles occupés et de l'environnement bâti virtuel.
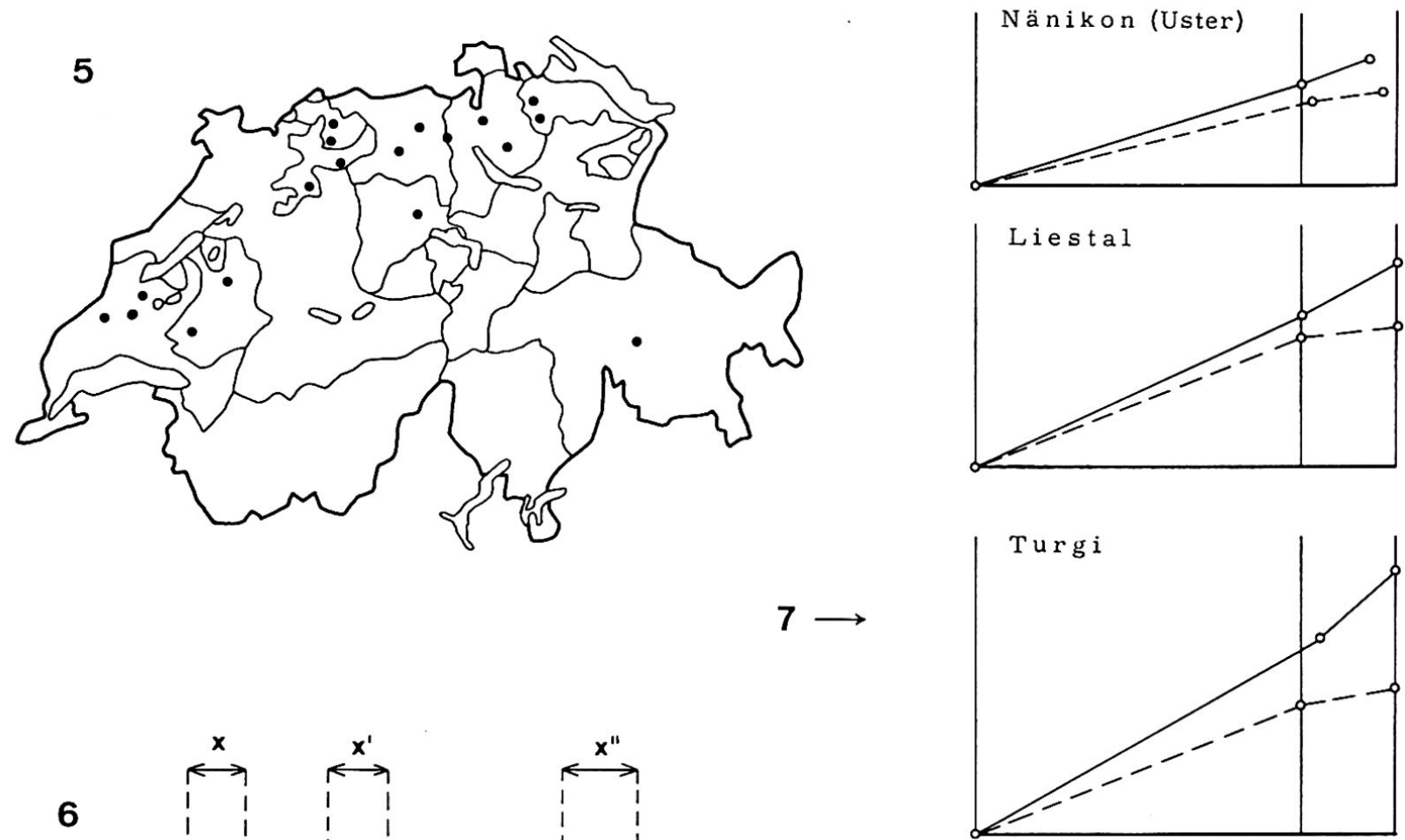

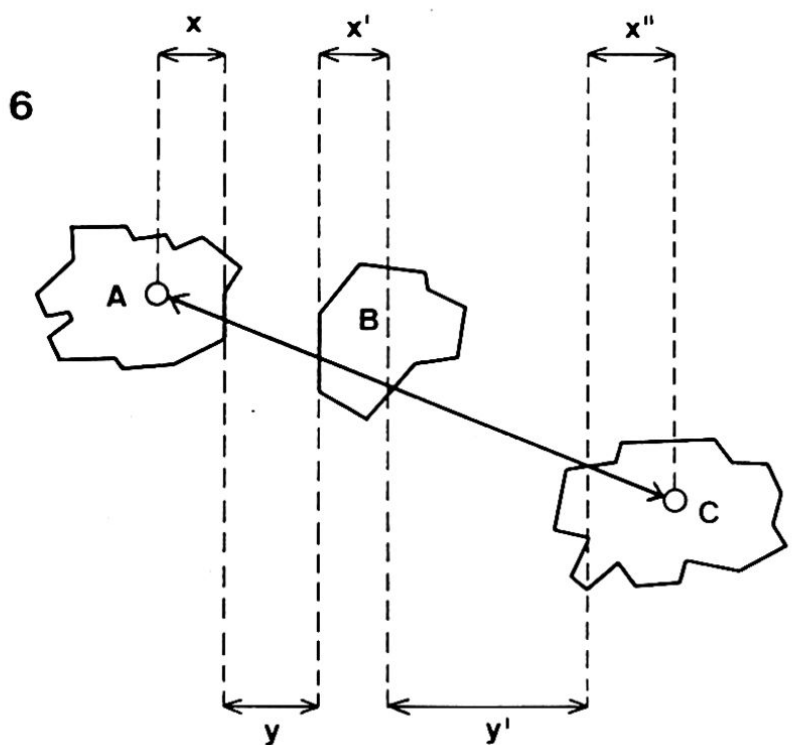

Tous les espaces construits supérieurs aux petits hameaux sont inscrits à l'intérieur d' un périmètre. Mais tous ne constituent pas, pour autant, des centres. Dans l'exemple ci-dessus, B correspond à un lotissement résidentiel.

L' environnement bâti virtuel, le long de l'itinéraire A C, est égal à : $\frac{x+x^{\prime}+x^{\prime \prime}}{y+y^{\prime}}$
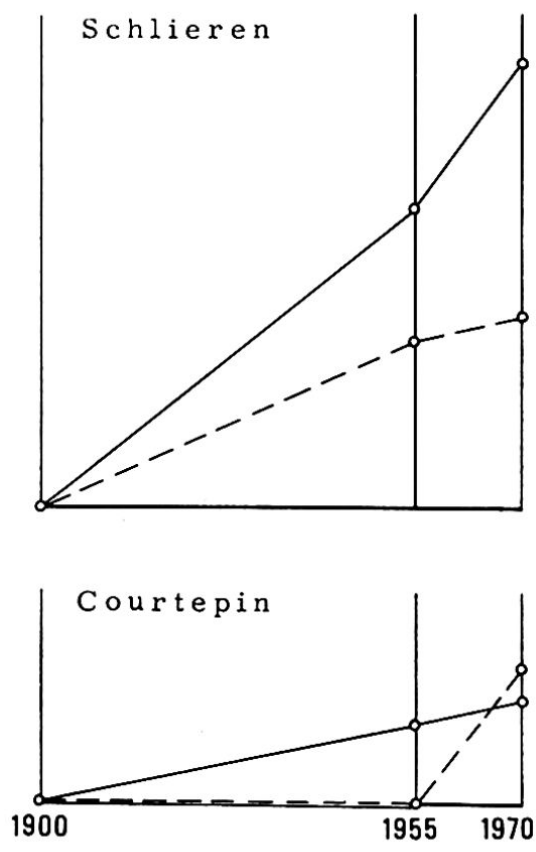